\title{
Adrenal insufficiency is seen in more than one-third of patients during ongoing low-dose prednisolone treatment for rheumatoid arthritis
}

\author{
Stina Willemoes Borresen ${ }^{1,2}$, Marianne Klose ${ }^{1}$, Bo Baslund ${ }^{2,3}$, \\ Åse Krogh Rasmussen ${ }^{1,2}$, Linda Hilsted ${ }^{2,4}$, Lennart Friis-Hansen ${ }^{5}$, Henning Locht ${ }^{6}$, \\ Annette Hansen ${ }^{7}$, Merete Lund Hetland ${ }^{2,8}$, Magnus Christian Lydolph ${ }^{9}$ and \\ Ulla Feldt-Rasmussen ${ }^{1,2}$
}

${ }^{1}$ Department of Medical Endocrinology, Copenhagen University Hospital, Rigshospitalet, Copenhagen, Denmark, ${ }^{2}$ Department of Clinical Medicine, Faculty of Health and Medical Sciences, University of Copenhagen, Copenhagen, Denmark, ${ }^{3}$ Center of Rheumatology and Joint Diseases, ${ }^{4}$ Department of Clinical Biochemistry, Copenhagen University Hospital, Rigshospitalet, Copenhagen, Denmark, ${ }^{5}$ Department of Clinical Biochemistry, Copenhagen University Hospital, Nordsjællands Hospital, Hillerød, Denmark, ${ }^{6}$ Center of Rheumatology and Joint Diseases, Copenhagen University Hospital, Frederiksberg Hospital, Frederiksberg, Denmark, ${ }^{7}$ Center of Rheumatology and Joint Diseases, Copenhagen University Hospital, Gentofte Hospital, Gentofte, Denmark, ${ }^{8}$ Center of Rheumatology and Joint Diseases, Copenhagen University Hospital, Rigshospitalet, Glostrup, Denmark, and ${ }^{9}$ Department of Autoimmunology and Biomarkers, Statens Serum Institut, Copenhagen, Denmark

Correspondence should be addressed to $U$ Feldt-Rasmussen Email ufeldt@rh.dk

\begin{abstract}
Objective: Patients receiving long-term glucocorticoid treatment are at risk of developing adrenal insufficiency during treatment. We investigated the prevalence of prednisolone-induced adrenal insufficiency in the particular clinical situation where patients receive ongoing low-dose ( $5 \mathrm{mg} /$ day) prednisolone treatment, a dose by itself too low to cover glucocorticoid needs during stress.

Design and methods: Cross-sectional study in 42 patients with rheumatoid arthritis (29 women, aged 36-86 years) treated with $5 \mathrm{mg}$ prednisolone/day, who had received prednisolone for $\geq 6$ months (median: 66, range:

6-444 months). Adrenal function was evaluated by a $250 \mu \mathrm{g}$ Synacthen test performed after mean $48.7 \mathrm{~h}$ prednisolone pause. Local assay-specific cut-off for normal adrenal function was P-cortisol $\geq 420 \mathrm{nmol} / \mathrm{L} 30 \mathrm{~min}$ after Synacthen injection.

Results: Overall, 20 of the 42 patients (48\%, 95\% Cl: 33-62\%) had an insufficient adrenal response to the Synacthen test. Including only patients who had not received concomitant treatment with any other glucocorticoid formulas within the last 3 months, 13 of 33 patients (39\%, 95\% Cl: 25-56\%) had an insufficient response. Adrenocorticotrophic hormone $(\mathrm{ACTH})$ concentrations were generally low and anti-adrenal antibodies were negative indicating secondary adrenal insufficiency as the most likely diagnosis. There was no correlation between duration of treatment and $30 \mathrm{~min}$ P-cortisol $(P=0.62)$. Adrenal function did not depend on sex or seropositivity of rheumatoid arthritis.

Conclusion: We demonstrate a high prevalence of adrenal insufficiency during ongoing low-dose prednisolone treatment. The results urge to increase focus on the condition to ensure identification and correct management of insufficient patients during stress and withdrawal. Strategies for adrenal function evaluation during ongoing low-dose glucocorticoid treatment need to be established.
\end{abstract}

() 2017 European Society of Endocrinology Printed in Great Britain
European Journal of

Endocrinology

(2017) 177, 287-295 


\section{Introduction}

Glucocorticoids are used in the treatment of a variety of inflammatory and autoimmune diseases, seldom with any doubt about the clinical indication and effect, but often with many side effects. Glucocorticoid-induced adrenal insufficiency is a potentially life-threatening side effect as it renders the patient unable to produce an adequate cortisol response to stress. Clinically relevant hypocortisolism can occur after withdrawal from glucocorticoid treatment, but also during ongoing treatment if there is a mismatch between glucocorticoid requirements to overcome stress and the sum of the endogenous cortisol production capacity and exogenous glucocorticoid intake. High-dose prednisolone treatment often ensures sufficient glucocorticoid intake to overcome most stressful situations, but low-dose prednisolone treatment might not. In individuals with normal adrenal function cortisol production varies from 5 to $10 \mathrm{mg} / \mathrm{m}^{2}$ surface area/day (equivalent to an oral hydrocortisone dose of $15-25 \mathrm{mg}$ /day in adults) in unstressful conditions (1) to an increased response to major stress of up to $75-150 \mathrm{mg}$ cortisol/day (2). For patients in low-dose prednisolone treatment, $5 \mathrm{mg} /$ day is equivalent to $20 \mathrm{mg} /$ day of hydrocortisone. If the adrenal function is suppressed these patients are thus not sufficiently covered during stress by the prednisolone treatment itself.

Glucocorticoid-induced adrenal insufficiency is probably underestimated in clinical practice $(3,4)$. In rheumatoid arthritis, low-dose prednisolone treatment for 2 years in addition to disease-modifying anti-rheumatic drugs reduces joint destruction and increases disease remission (5), and is thus widely used both as initial, but also maintenance therapy $(6,7)$. It is currently not recommended to evaluate adrenal function routinely during the course of glucocorticoid treatment (8), which potentially leaves a large group of patients with longlasting adrenal insufficiency, who are not adequately informed about the risk and treatment of adrenal insufficiency during intercurrent stress.

We aimed to assess the prevalence of glucocorticoidinduced adrenal insufficiency in patients with rheumatoid arthritis treated with $5 \mathrm{mg}$ prednisolone daily to investigate whether adrenal insufficiency is a frequent and thereby clinically significant problem during long-term low-dose prednisolone treatment.

\section{Subjects and methods}

\section{Participants}

Patients with rheumatoid arthritis were retrieved from four rheumatology departments located in the greater Copenhagen area in Denmark. The diagnosis of rheumatoid arthritis was based on the American Rheumatism Association 1987 classification (9). Patients treated with $5 \mathrm{mg}$ prednisolone daily and in continuous treatment for a minimum of 6 months were considered for inclusion. Further inclusion criteria were: age $\geq 18$ years and Caucasian ethnicity. In total 127 consecutive patients were identified. Patients were excluded if they were unable to provide a written informed consent $(n=9)$, pregnant $(n=1)$, unwilling to discontinue any estrogen treatment 6 weeks prior to a Synacthen test $(n=2)$ or had other major confounding diseases contraindicating $48 \mathrm{~h}$ of prednisolone discontinuation $(n=2)$.

In total 113 patients were eligible for inclusion, and $54(48 \%)$ of these were willing to participate.

The patients were recruited as part of a larger study (ClinicalTrials.gov no. NCT01411046), and were screened for four specific polymorphisms of the glucocorticoid receptor gene (NR3C1) and grouped accordingly before being invited to a Synacthen test. Thus, both patients with or without these polymorphisms were invited to a Synacthen test, but patients with a mixed hetero- and homozygote genotype were excluded ( $n=12$ of $54(22 \%$ of patients)) due to the overall study protocol. In total, 42 patients underwent assessment of adrenal function. Disease history and data on history of treatment with prednisolone and other glucocorticoid containing formulations were obtained from medical records and confirmed by patient interview.

The protocol was approved by the local Ethics Committee (J.nr. H-4-2011-051) and the Data Protection Agency (J.nr. 2007-58-0015, local 30-0580). All participants gave written informed consent before enrolment.

\section{Adrenal function assessment}

Adrenal function was evaluated by a Synacthen (corticotropin) stimulation test performed in the morning, starting between 08:00 and 10:30h, after an overnight fast and a prednisolone pause of approximately 
$48 \mathrm{~h}$ (depending on individual dose administration time). A bolus of $250 \mu \mathrm{g}$ tetracosactid (Synacthen; Sigma-tau, Industrie Farmaceutiche Riunite S.p.A., Rome, Italy) was administered i.v. in a large forearm vein. P-cortisol was measured before and $30 \mathrm{~min}$ after Synacthen injection. All Synacthen tests were performed at the tertiary referral center, Department of Medical Endocrinology, Copenhagen University Hospital, Rigshospitalet, Denmark. After the Synacthen test patients continued the prednisolone treatment.

\section{Assays and cut-offs}

P-cortisol was measured by the Elecsys Cortisol II immunoassay (Roche GmbH, Germany) (10) on Cobas 8000 e602 module platform. Elecsys Cortisol II is a new generation automated cortisol immunoassay with increased specificity due to use of monoclonal antibodies and standardization against mass spectrometry with an expected decrease in cortisol concentrations by approximately $20 \%$ compared to older generation cortisol assays. Maximal coefficient of variation $\left(\mathrm{CV}_{\max }\right)$ in the assay was $8 \%$. Prednisolone has cross-reactivity in the assay of $8 \%$. Samples were analyzed in few series.

Cut-off for normal cortisol response to the Synacthen test was validated locally for the new assay (11) and defined as the 25 th percentile $-1.96^{*}$ S.E. of the $30 \mathrm{~min}$ cortisol response to an $250 \mu \mathrm{g}$ Synacthen test in 100 healthy volunteers as previously described (12). This derived a cut-off of $420 \mathrm{nmol} / \mathrm{L}$ which is lower than the commonly recommended $500 \mathrm{nmol} / \mathrm{L}$, but similar to the cut-off previously suggested for mass spectrometry (13).

Plasma concentrations of adrenocorticotrophic hormone (ACTH) and anti-adrenal antibodies were measured at baseline to distinguish patients with primary (autoimmune) adrenal insufficiency.

ACTH in plasma was analyzed by a sandwich chemiluminescent immunometric method using Siemens' reagents on an Immulite 2000 platform (Siemens $\mathrm{GmbH}$, Germany) with $\mathrm{CV}_{\max }$ of $10 \%$. Adrenal cortex antibodies were measured by indirect immunofluorescence microscopy on primate adrenal sections (INOVA Diagnostics, San Diego, CA, USA). Briefly, one drop of patient sample diluted 1:10 in phosphate-buffered saline (PBS) was applied to each well on substrate slides. After 30 min incubation in a moist chamber, the slides were washed in PBS and placed in a glass chamber with PBS for $5 \mathrm{~min}$. Excess PBS was shaken off the slides and one drop of fluorescein isothiocyanate (FITC) anti-human IgG conjugate (DAKO \#F0202) was added before incubation for $30 \mathrm{~min}$. Following another washing step slides were analyzed in a fluorescence microscope at $495 \mathrm{~nm}$ exciter. A sample was considered positive if the specific staining was observed to be greater than a negative control.

\section{Statistical analysis}

Categorical data are presented as number, $n$ (\%), continuous data as mean ( \pm s.D.) if normally distributed and otherwise as median (range). Age, prednisolone pause, baseline P-cortisol and 30 min P-cortisol followed a Gaussian distribution, whereas duration of treatment and ACTH concentrations were log Gaussian distributed. Ninety-five percent confidence intervals for proportions were calculated by the Wilson Score interval formula. Comparison between groups was made by independent t-test for normally distributed continuous data and Chisquare test for categorical data, since data were equally distributed among cells. Correlation analyses were performed to assess the association between $30 \mathrm{~min}$ P-cortisol and duration of treatment, results presented with Pearson's correlation coefficient. The prevalence of adrenal insufficiency and mean $30 \mathrm{~min}$ P-cortisol were compared between groups stratified by sex and presence of IgM rheumatoid factor in rheumatoid arthritis. All statistical analyses were performed by SAS version 9.4 (SAS institute Inc., Cary, NC, USA). A difference was considered significant when $P<0.05$.

\section{Results}

\section{Patient characteristics}

Of the 127 identified patients, gender and seropositivity of rheumatoid arthritis did not differ between 42 patients who participated in the study and 85 patients who were either excluded $(n=26)$ or unwilling to participate $(n=59)$. Patients in the study were generally younger (mean age 65 years (s.D. 14) vs 71 years (s.D. 13), $P=0.03$ ).

The characteristics of the study population at the time of the Synacthen test are shown in Table 1 . The 42 patients had been treated with prednisolone for a median of 66 months (range: 6-444 months). All patients were treated with $5 \mathrm{mg}$ prednisolone/day until pausing mean 48.7 h (range: 36-96h) before the Synacthen test. Within the last 6 months before the Synacthen test, 6 (14\%) patients had received prednisolone doses above $5 \mathrm{mg}$ and $2(4.8 \%)$ patients had received doses below $5 \mathrm{mg}$, but none within the last month. Nine (21\%) patients were in addition treated with locally applied glucocorticoids, 
Table 1 Patient characteristics at the time of the Synacthen test. Data are presented as number (\%) or mean (s.D.)/median (range).

\begin{tabular}{|c|c|}
\hline & All patients \\
\hline$n$ & 42 \\
\hline Sex (men) & $13(31 \%)$ \\
\hline Seropositive rheumatoid arthritis & $23(55 \%)$ \\
\hline Age (years) & $65.3(12.6)$ \\
\hline Duration of therapy (months) & $66(6-444)$ \\
\hline Prednisolone pause before Synacthen test $(\mathrm{h})$ & $48.7(10.2)$ \\
\hline \multicolumn{2}{|l|}{ Prednisolone dose $>5 \mathrm{mg} /$ day } \\
\hline 1 month before Synacthen test & $0(0 \%)$ \\
\hline 2-6 months before Synacthen test & $6(14 \%)^{a}$ \\
\hline \multicolumn{2}{|l|}{ Prednisolone doses $<5 \mathrm{mg} /$ day } \\
\hline 3-6 months before Synacthen test & $2(4.8 \%)^{b}$ \\
\hline $\begin{array}{l}\text { Concomitant treatment with other } \\
\text { glucocorticoids }\end{array}$ & $9(21 \%)$ \\
\hline Intra-articular injections & $4(9.5 \%)^{c}$ \\
\hline Intra-muscular injections & $4(9.5 \%)^{d}$ \\
\hline Glucocorticoid containing cream & $1(2.4 \%)^{\mathrm{e}}$ \\
\hline
\end{tabular}

a 7.5-15 mg (median $7.5 \mathrm{mg}$ ) for $1-5$ months (median 2.5 months); ${ }^{\text {b }} 2.5 \mathrm{mg}$ for $0.75-1$ months; ' paused 2, 5, 6 and 8 weeks respectively; ${ }^{d}$ paused 1, 4, 5 and 6 weeks respectively; ${ }^{e}$ paused several weeks.

which were paused as long time before the Synacthen test as possible (Table 1).

\section{Adrenal function}

Overall, 20 of the 42 patients (48\%, 95\% CI: 33-62\%) had an insufficient adrenal response to the Synacthen test (Fig. 1). Including only patients who had not received concomitant treatment with any other glucocorticoid containing formulas within the last 3 months before the Synacthen test 13 of the 33 patients (39\%, 95\% CI: 25-56\%) had an insufficient adrenal response to the Synacthen test. Of the nine patients who had received concomitant intra-articular injections, intra-muscular injections or treatment with glucocorticoid containing cream respectively, 4/4, 3/4 and $0 / 1$ respectively had an insufficient response to the Synacthen test. Three of the six $(50 \%)$ patients, who had received prednisolone doses higher than $5 \mathrm{mg}$ and both patients who had received doses below $5 \mathrm{mg}$ within the last 6 months before the Synacthen test had adrenal insufficiency.

There was no correlation between duration of treatment and $30 \mathrm{~min}$ P-cortisol ( $P=0.62, \quad r=-0.08)$. Neither sex nor presence of rheumatoid factor were associated with adrenal function ( $t$-test for comparison of mean 30 min P-cortisol $P=0.32$ and $P=0.42$ respectively, $\chi^{2}$ for adrenal insufficiency $P=0.52$ and $P=0.43$ respectively).

ACTH concentrations obtained in 41 (97\%) patients were generally low within the reference range with a

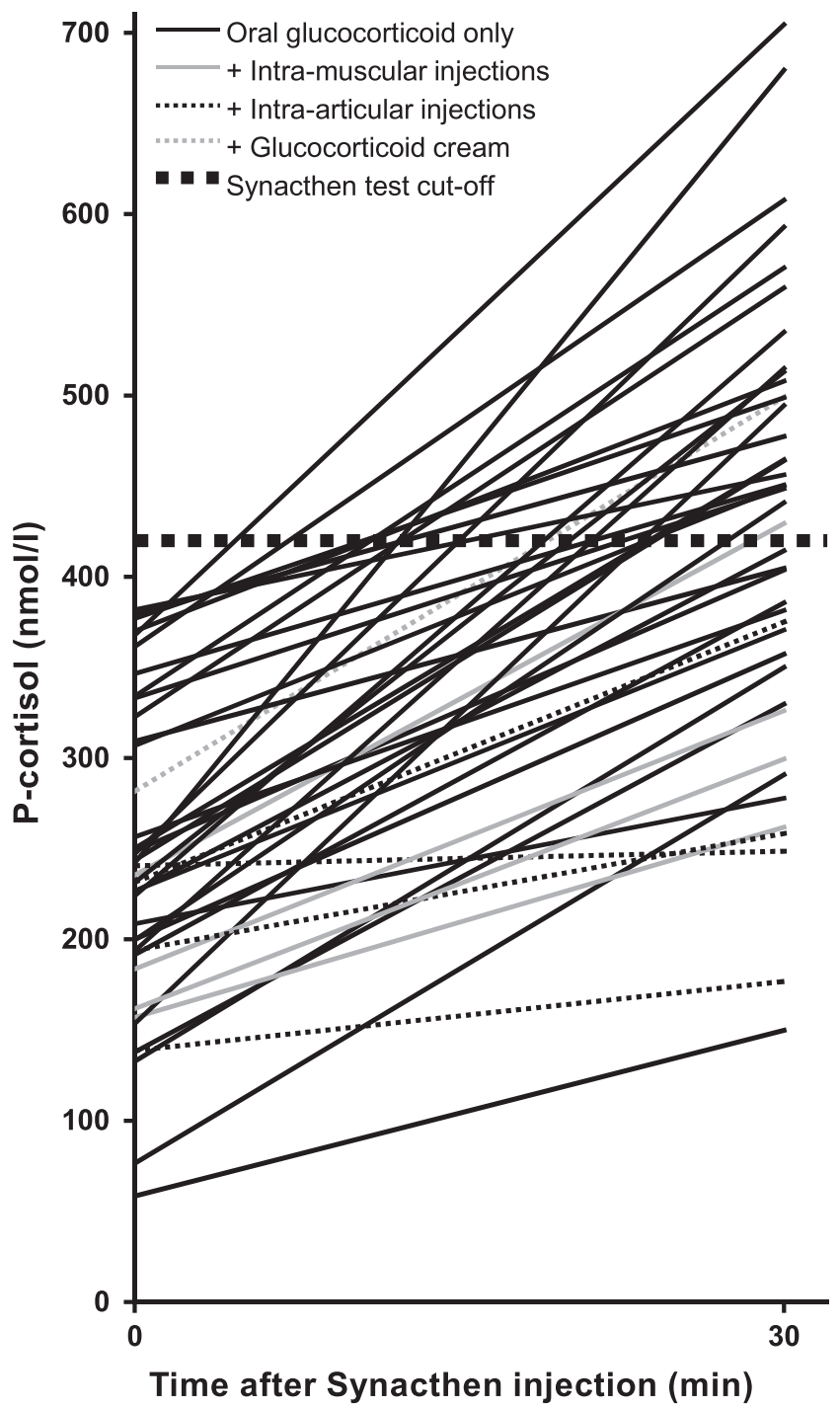

Figure 1

Spaghetti plot of P-cortisol levels at baseline and $30 \mathrm{~min}$ after $250 \mu \mathrm{g}$ Synacthen injection in patients treated with $5 \mathrm{mg}$ prednisolone/day. Each line represents one patient. Black lines represent patients who did not concomitantly receive treatment with other glucocorticoid formulas. Patients who received concomitant treatment with other glucocorticoid formulas within the last 3 months before the Synacthen test are presented by grey lines (intra-muscular injections), dotted black lines (intra-articular injections) or dotted grey line (glucocorticoid containing cream). Cut-off for insufficient adrenal function: $30 \mathrm{~min}$ P-cortisol $<420 \mathrm{nmol} / \mathrm{L}$ is marked by a dotted horizontal line. Overall $48 \%$ of the patients had adrenal insufficiency. Including only patients who had not received concomitant treatment with any other glucocorticoid formulas within the last 3 months $39 \%$ had adrenal insufficiency. 


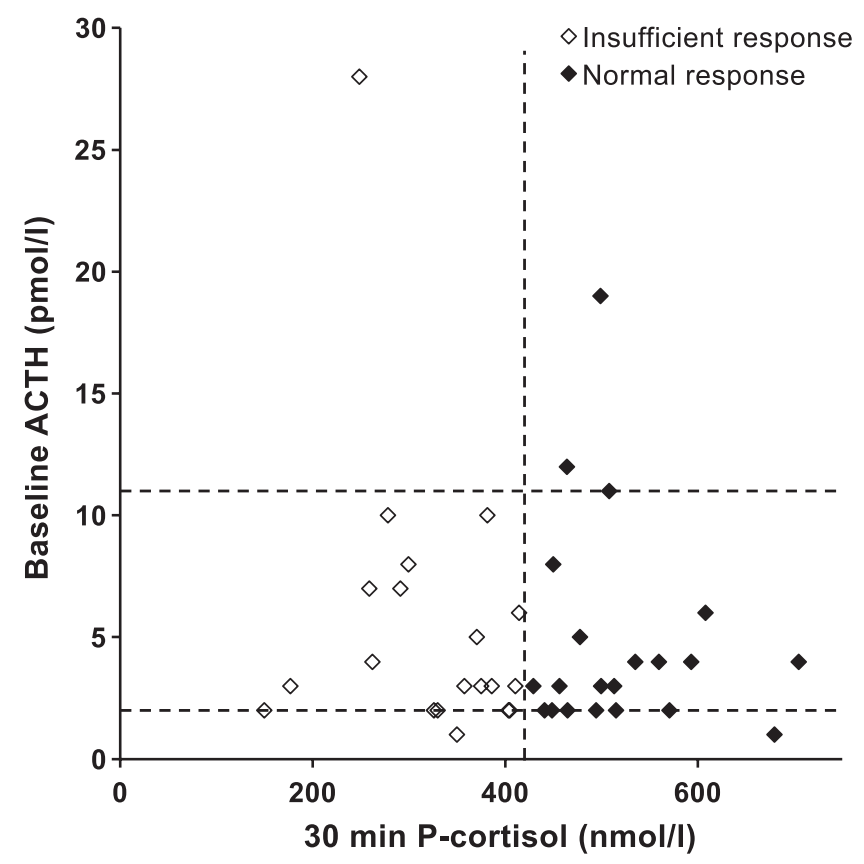

Figure 2

ACTH levels marked on the $y$-axis and P-cortisol 30 min after $250 \mu \mathrm{g}$ Synacthen injection on the $x$-axis. The ACTH reference range is marked within horizontal dotted lines. $\diamond$ represents patients with insufficient response to the Synacthen test, $\checkmark$ represents patients with normal response. Synacthen test cut-off is marked by a vertical dotted line.

median of $3 \mathrm{pmol} / \mathrm{L}$ (range $<1-28 \mathrm{pmol} / \mathrm{L}$ ) (Fig. 2). Antiadrenal antibodies were analyzed in 38 (90\%) patients and were negative in all of these patients.

\section{Discussion}

In the present study, we found adrenal insufficiency in more than one-third of the patients treated with $5 \mathrm{mg}$ prednisolone daily. Higher occurrence was observed in the total cohort, which also included patients who had received concomitant treatment with other glucocorticoid formulas. Glucocorticoid-induced adrenal insufficiency is often believed to occur in patients who have been treated with more than the equivalent of $7.5 \mathrm{mg} /$ day prednisolone $(8,14,15,16)$ and since patients in highdose prednisolone treatment are sufficiently covered during most stress, the dominating clinical focus on glucocorticoid-induced adrenal insufficiency has been on adrenal insufficiency after withdrawal from glucocorticoid treatment. We here show that glucocorticoid-induced adrenal insufficiency is also a problem during ongoing low-dose prednisolone treatment. Our study differs from previous studies in that it only included patients receiving $5 \mathrm{mg}$ prednisolone/day. This was chosen to investigate the prevalence of adrenal insufficiency during ongoing prednisolone treatment in the particular situation, where the prednisolone dose is too low to cover extra needs during stress. The somewhat higher prevalence of $78 \%$ in the nine patients, who received concomitant treatment with glucocorticoid containing cream, intra-muscular or intra-articular glucocorticoid injections stresses that these administration routes are important contributors to adrenal suppression. Adrenal insufficiency can occur after all locally applied glucocorticoids $(4,17)$, and high prevalence has especially been demonstrated after intraarticular glucocorticoid injections $(4,18,19,20,21)$ and in patients using multiple glucocorticoid forms concomitantly (4). Adrenal suppression after a single intra-articular glucocorticoid injection has been shown to last up to 4 weeks $(19,20,21)$. In the present study, the Synacthen test was performed $\geq 4$ weeks after an intra-articular or -muscular glucocorticoid injection in all but two patients (Table 1). At the time of the Synacthen test the concomitant glucocorticoid treatment did not contribute to cover patients' glucocorticoid need during stress.

All patients in our cohort had been treated with a stable dose of $5 \mathrm{mg}$ prednisolone/day the last month leading up to the Synacthen test and $81 \%$ of patients for more than 6 months. Six patients had received higher doses at some point within the 6 months before the Synacthen test. Three (50\%) of these had adrenal insufficiency and inclusion of these patients therefore did not affect the overall prevalence in the study. The patient cohort was representative of patients with rheumatoid arthritis in lowdose prednisolone treatment where variations in disease activity lead to short periods of increased prednisolone dose or intra-articular or -muscular corticosteroid injections. Likewise, the cohort is comparable to other patient populations in glucocorticoid treatment where increased disease activity is treated with increased glucocorticoid intake (increased dose or additional formula). For these populations the overall prevalence of $48 \%$ might be a relevant estimate. For patient populations seldom receiving concomitant treatment with other glucocorticoid formulas the reported prevalence of 39\% might be more comparable. Our findings are similar to studies of patients receiving more varying (22) or slightly higher glucocorticoid doses $(23,24)$.

We found no association between adrenal function and duration of prednisolone treatment. However, it is possible that such an association would be clearer 
for shorter durations than we have investigated in the present study. The risk of adrenal insufficiency generally increases with increased duration of glucocorticoid treatment $(4,24)$, but glucocorticoid-induced adrenal suppression is at the same time associated with a substantial individual variation with less strong association to glucocorticoid dose or duration of treatment $(3,22,25)$.

The study has confirmed a strong suspicion that adrenal insufficiency is highly prevalent during ongoing low-dose prednisolone treatment, which was the aim of the study. As patients already suffered from one autoimmune disease (rheumatoid arthritis) we aimed at excluding primary adrenal insufficiency. Since ACTH concentrations were generally low and anti-adrenal antibodies were negative this was very unlikely, and the diagnosis of secondary, glucocorticoid-induced adrenal insufficiency was considered accurate.

For reliable assessment of adrenal function, it is generally agreed that a stimulation test is necessary (3, $26,27,28)$. In the present study, we used the standarddose $250 \mu \mathrm{g}$ Synacthen test as it is simple and safe and correlates well with the insulin tolerance test $(29,30)$. Some $(31,32,33)$ but not all $(27,28,34)$ prefer the lowdose $1 \mu \mathrm{g}$ Synacthen test over the standard-dose $250 \mu \mathrm{g}$ Synacthen test. The low-dose test has been found to be more sensitive in detecting mild adrenal insufficiency as the stimulation is at a more physiological level $(31,32)$, but technical details can influence its accuracy, resulting in reduced specificity $(32,34,35)$. There is no published consensus on which test is preferred.

The use of the $250 \mu \mathrm{g}$ Synacthen test in our study minimized the risk of false-positive test results.

P-cortisol measurement was performed with a new generation assay, the Roche Elecsys Cortisol II assay, performing more specific (and thus lower) cortisol measurements than the older Roche Elecsys Cortisol assay. All cortisol samples were frozen and analyzed in few series. Local assay-specific cut-off for normal adrenal function was validated for the new assay (11) based on Synacthen tests performed in previously described healthy controls (12), minimizing the risk of methodological bias. The cross-reactivity of prednisolone in the Roche Elecsys Cortisol II assay is very low (8\% (10)) compared with the older Roche Elecsys Cortisol assays (up to $171 \%$ $(36,37)$ ). The prednisolone pause of mean $48.7 \mathrm{~h}$ (range 36-96h) comprised 14-25 plasma half-lives (range 9-48 half-lives) for prednisolone (38) and was chosen to minimize the risk of measuring falsely-high cortisol levels thus underestimating adrenal insufficiency due to cross-reactivity weighted against the safety aspect of the prednisolone pause for the patients.

The reported prevalence in this study may be surprising to clinicians who could argue that symptomatic patients would nevertheless be identified. However, in a metaanalysis only 10 of 98 patients with glucocorticoid-induced adrenal insufficiency reported symptoms of adrenal insufficiency, concluding that the diagnosis would have been missed in 88 patients if only symptomatic patients underwent assessment of adrenal function (4). Adrenal insufficiency most often presents with very unspecific symptoms. Patients may present with symptoms such as fatigue, loss of energy, muscle and joint pain which could result from increased disease activity of rheumatoid arthritis, but could as well relate to adrenal insufficiency. Adrenal insufficiency should especially be suspected when other signs and symptoms of disease activity of the rheumatoid arthritis (C-reactive protein, joint swelling, etc.) do not reflect the patients' symptoms. It is a general clinical observation that prednisolone tapering is very difficult in many patients with rheumatoid arthritis and other rheumatologic diseases (39). Two patients in our study had failed attempts to taper the prednisolone dose shortly before enrolment in the study. They were tapered to doses below $5 \mathrm{mg}$, but had to increase the dose again after 3-4 weeks due to flare up of symptoms despite the absence of clinical signs of disease activity. Both of these patients were found to have adrenal insufficiency. During the 48-h prednisolone pause before the study Synacthen test several patients experienced an increase in muscle and joint pain and some felt generally unwell. Although not systematically registered it was a clinical observation that there was a connection between having notable symptoms during the prednisolone pause and a reduced response to the Synacthen test. All patients were informed to contact the department or simply resume prednisolone and postpone the Synacthen test if they felt unwell, but none chose this option.

Also signs and symptoms of an acute adrenal crisis are mostly unspecific and can be confused with an exacerbation of the underlying disease or symptoms of the triggering stressful event such as gastroenteritis or influenza-like illnesses. Thus, there is a high risk of misclassification of adrenal crises in glucocorticoidtreated patients without verified adrenal insufficiency and retrospective studies investigating the incidence of glucocorticoid-induced adrenal crises cannot account for all the dark numbers of incorrectly classified and coded crises (40). The incidence of glucocorticoid-induced adrenal crisis is thus difficult to clarify. In 28 patients 
with an established diagnosis of glucocorticoid-induced adrenal insufficiency the reported incidence of adrenal crises was 15 per 100 patient-years (41). This was higher than the incidence of 5.2 and 3.6 adrenal crisis per 100 patient-years found in patients with primary $(n=111)$ and secondary (other than glucocorticoid-induced) $(n=319)$ adrenal insufficiency respectively (41). Similar, a prospective study has reported an incidence of 8.3 adrenal crises per 100 patient-years and associated mortality in approximately $6 \%$ of adrenal crises for patients with known primary $(n=221)$ or secondary (other than glucocorticoid-induced) $(n=202)$ adrenal insufficiency (42). Overall, these data suggest that adrenal crisis can occur in patients during ongoing low-dose prednisolone treatment who have (severely) suppressed adrenal function.

Our results call for increased focus on the adrenal function during long-term low-dose prednisolone treatment. We argue that all patients receiving glucocorticoid treatment should be informed about the risk of adrenal insufficiency and should carry a steroid emergency card $(43,44)$. Whether insufficient patients on ongoing low-dose prednisolone treatment could benefit from receiving supplemental glucocorticoid doses during intercurrent illness and stress has not been shown, but it is standard procedure in all other types of adrenal insufficiency.

Our findings make a case for routine evaluation of adrenal function in patients on ongoing low-dose glucocorticoid treatment, but there are some concerns in regard to defining correct timing and frequency of such routine evaluation and the increased cost and workload. It might not be feasible everywhere. Future perspectives such as using baseline morning P-cortisol measurements as an initial diagnostic screening tool should be further explored $(45,46)$.

If the activity of the underlying disease for which the prednisolone is prescribed allows prednisolone tapering and potentially withdrawal the need for daily replacement therapy should be considered. Hydrocortisone is preferred for replacement therapy over prednisolone as its shorter half-life enables a more physiological mimic of the normal circadian rhythm of cortisol secretion $(47,48)$ with low nightly levels improving chances for adrenal recovery. Adrenal function evaluation and treating the adrenal insufficiency with physiological doses of hydrocortisone might enable reduction or even withdrawal of prednisolone, thereby reducing other prednisolone-induced side effects such as osteoporosis, diabetes and hypertension.
In conclusion, we have investigated secondary adrenal insufficiency in the particular clinical situation where patients receive ongoing prednisolone treatment in a dose that in itself is too low to cover extra glucocorticoid needs during stress. We found that more than one-third of the patientshad adrenal insufficiency. Aslow-doseglucocorticoid treatment is widely used, not only in rheumatoid arthritis, but in many different conditions, the prevalence is alarming and many patients might have unidentified adrenal insufficiency. If future studies were to find the same benefit from hydrocortisone replacement strategy in patients with glucocorticoid induced adrenal insufficiency as for other patients with adrenal insufficiency, clinical management guidelines would have to be implemented for this patient group. Correct management could potentially reduce adrenal crisis, improve patients' quality of life and may help facilitate tapering from prednisolone treatment reducing overall steroid side-effects.

Declaration of interest

The authors declare that there is no conflict of interest that could be perceived as prejudicing the impartiality of this clinical study.

\section{Funding}

The study was supported by unrestricted grants from: The Eva Maduras Foundation, The Research Foundation of Copenhagen University Hospital, Rigshospitalet (R110-A4402), The Danish Rheumatism Association (R141-A4022) and The Research Foundation of The Capital Region of Denmark. U F-R's research salary was partly funded by Arvid Nilsson's Fund, partly by Novo Nordic Foundation as an unrestricted research grant (application number 8005 and 14266).

Author contribution statement

S W Borresen made primary contributions to data collection and analysis, interpretation of results, and writing of the manuscript. B Baslund, S W Borresen, U Feldt-Rasmussen, L Friis-Hansen, L Hilsted, M Klose and $\AA \mathrm{K}$ Rasmussen contributed to the study conception and design. B Baslund, S W Borresen, U Feldt-Rasmussen, L Friis-Hansen, A Hansen, M L Hetland, L Hilsted, M Klose, H Locht, M C Lydolph and $\AA$ K Rasmussen contributed to data collection/analyses. All authors contributed to interpretation of results, all revised the manuscript critically for important intellectual content and all approved the final manuscript. $\mathrm{S} \mathrm{W}$ Borresen is the guarantor.

\section{Acknowledgements}

The authors thank laboratory technician Casper Kok for his excellent technical assistance and medical students Tanja Rasmussen and Helin Shehab for their excellent help in collecting data.

\section{References}

1 Esteban NV, Loughlin T, Yergey AL, Zawadzki JK, Booth JD, Winterer JC \& Loriaux DL. Daily cortisol production rate in man determined 
by stable isotope dilution/mass spectrometry. Journal of Clinical Endocrinology and Metabolism 199172 39-45. (doi:10.1210/ jcem-72-1-39)

2 Kehlet H. A rational approach to dosage and preparation of parenteral glucocorticoid substitution therapy during surgical procedures. A short review. Acta Anaesthesiologica Scandinavica 197519 260-264. (doi:10.1111/j.1399-6576.1975.tb05182.x)

3 Dinsen S, Baslund B, Klose M, Rasmussen AK, Friis-Hansen L, Hilsted L \& Feldt-Rasmussen U. Why glucocorticoid withdrawal may sometimes be as dangerous as the treatment itself. European Journal of Internal Medicine 201324 714-720. (doi:10.1016/j.ejim.2013.05.014)

4 Broersen LH, Pereira AM, Jorgensen JO \& Dekkers OM. Adrenal insufficiency in corticosteroids use: systematic review and metaanalysis. Journal of Clinical Endocrinology and Metabolism 2015100 2171-2180. (doi: 10.1210/jc.2015-1218).

5 Svensson B, Boonen A, Albertsson K, van der Heijde D, Keller C $\&$ Hafstrom I. Low-dose prednisolone in addition to the initial disease-modifying antirheumatic drug in patients with early active rheumatoid arthritis reduces joint destruction and increases the remission rate: a two-year randomized trial. Arthritis and Rheumatism 200552 3360-3370. (doi:10.1002/art.21298)

6 Bijlsma JW, van der Goes MC, Hoes JN, Jacobs JW, Buttgereit F \& Kirwan J. Low-dose glucocorticoid therapy in rheumatoid arthritis: an obligatory therapy. Annals of the New York Academy of Sciences 2010 1193 123-126. (doi:10.1111/j.1749-6632.2009.05342.x)

7 Hoes JN, Jacobs JW, Boers M, Boumpas D, Buttgereit F, Caeyers N, Choy EH, Cutolo M, Da Silva JA, Esselens G et al. EULAR evidencebased recommendations on the management of systemic glucocorticoid therapy in rheumatic diseases. Annals of the Rheumatic Diseases 200766 1560-1567. (doi:10.1136/ard.2007.072157)

8 van der Goes MC, Jacobs JW, Boers M, Andrews T, Blom-Bakkers MA, Buttgereit F, Caeyers N, Cutolo M, Da Silva JA, Guillevin L et al. Monitoring adverse events of low-dose glucocorticoid therapy: EULAR recommendations for clinical trials and daily practice. Annals of the Rheumatic Diseases 201069 1913-1919. (doi:10.1136/ ard.2009.124958)

9 Arnett FC, Edworthy SM, Bloch DA, McShane DJ, Fries JF, Cooper NS, Healey LA, Kaplan SR, Liang MH, Luthra HS et al. The American Rheumatism Association 1987 revised criteria for the classification of rheumatoid arthritis. Arthritis and Rheumatism 198831 315-324. (doi:10.1002/art.1780310302)

10 Diagnostics R. Cortisol II Kit Insert. Mannheim: Roche Diagnostic, 2015.

11 Klose M, Hilsted L \& Feldt-Rasmussen U. Method-specific serum cortisol responses to the ACTH test: comparison of two generations of Roche automated immunoassays using polyclonal vs MABs. Endocrine Abstracts 201641 EP22. (doi: 10.1530/endoabs.41.EP22)

12 Klose M, Lange M, Rasmussen AK, Skakkebaek NE, Hilsted L, Haug E, Andersen M \& Feldt-Rasmussen U. Factors influencing the adrenocorticotropin test: role of contemporary cortisol assays, body composition, and oral contraceptive agents. Journal of Clinical Endocrinology and Metabolism 200792 1326-1333. (doi:10.1210/ jc.2006-1791)

13 El-Farhan N, Pickett A, Ducroq D, Bailey C, Mitchem K, Morgan N, Armston A, Jones L, Evans C \& Rees DA. Method-specific serum cortisol responses to the adrenocorticotrophin test: comparison of gas chromatography-mass spectrometry and five automated immunoassays. Clinical Endocrinology 201378 673-680. (doi:10.1111/ cen.12039)

14 Livanou T, Ferriman D \& James VH. Recovery of hypothalamopituitary-adrenal function after corticosteroid therapy. Lancet 19672 856-859. (doi:10.1016/S0140-6736(67)92592-5)

15 Stewart PM \& Krone NP. The adrenal cortex. In Williams Textbook of Endocrinology, edn 12th, p 517. Eds RH Williams \& S Melmed. Philidelphia: Elsevier/Saunders, 2011.
16 LaRochelle GE Jr, LaRochelle AG, Ratner RE \& Borenstein DG. Recovery of the hypothalamic-pituitary-adrenal (HPA) axis in patients with rheumatic diseases receiving low-dose prednisone. American Journal of Medicine 199395 258-264. (doi:10.1016/00029343(93)90277-V)

17 Borresen SW, Klose M, Rasmussen AK \& Feldt-Rasmussen U. Adrenal insufficiency caused by locally applied glucocorticoids-myth or fact? Current Medicinal Chemistry 201522 2801-2809. (doi:10.2174/092986 7322666150716113003)

18 Duclos M, Guinot M, Colsy M, Merle F, Baudot C, Corcuff JB \& Lebouc Y. High risk of adrenal insufficiency after a single articular steroid injection in athletes. Medicine and Science in Sports and Exercise 200739 1036-1043. (doi:10.1249/mss.0b013e31805468d6)

19 Habib G, Jabbour A, Artul S \& Hakim G. Intra-articular methylprednisolone acetate injection at the knee joint and the hypothalamic-pituitary-adrenal axis: a randomized controlled study. Clinical Rheumatology 201433 99-103. (doi:10.1007/s10067-0132374-4)

20 Habib G, Elias S, Abu-Elhaija M, Sakas F, Khazin F, Artul S, Jabbour A $\&$ Jabaly-Habib H. The effect of local injection of methylprednisolone acetate on the hypothalamic-pituitary-adrenal axis among patients with greater trochanteric pain syndrome. Clinical Rheumatology 2017 36 959-963. (doi:10.1007/s10067-016-3517-1)

21 Habib G, Zahran R, Najjar R, Badarny S, Jabbour A, Artul S, Hakim G \& Jabaly-Habib H. The effect of intra-articular injection of Diprospan at the knee joint on the hypothalamic-pituitary-adrenal axis. Swiss Medical Weekly 2015145 w14134. (doi:10.4414/smw.2015.14134)

22 Schlaghecke R, Kornely E, Santen RT \& Ridderskamp P. The effect of long-term glucocorticoid therapy on pituitary-adrenal responses to exogenous corticotropin-releasing hormone. New England Journal of Medicine 1992326 226-230. (doi:10.1056/NEJM199201233260403)

23 Kirwan JR, Hickey SH, Hallgren R, Mielants H, Bjorck E, Persson T \& Wollheim FA. The effect of therapeutic glucocorticoids on the adrenal response in a randomized controlled trial in patients with rheumatoid arthritis. Arthritis and Rheumatism 200654 1415-1421. (doi:10.1002/ art.21747)

24 Sacre K, Dehoux M, Chauveheid MP, Chauchard M, Lidove O, Roussel R \& Papo T. Pituitary-adrenal function after prolonged glucocorticoid therapy for systemic inflammatory disorders: an observational study. Journal of Clinical Endocrinology and Metabolism 201398 3199-3205. (doi:10.1210/jc.2013-1394)

25 Henzen C, Suter A, Lerch E, Urbinelli R, Schorno XH \& Briner VA. Suppression and recovery of adrenal response after short-term, high-dose glucocorticoid treatment. Lancet 2000355 542-545. (doi:10.1016/S0140-6736(99)06290-X)

26 Bornstein SR, Allolio B, Arlt W, Barthel A, Don-Wauchope A, Hammer GD, Husebye ES, Merke DP, Murad MH, Stratakis CA et al. Diagnosis and treatment of primary adrenal insufficiency: an endocrine society clinical practice guideline. Journal of Clinical Endocrinology and Metabolism 2016101 364-389. (doi:10.1210/jc.2015-1710)

27 Grossman AB. Clinical review\#: the diagnosis and management of central hypoadrenalism. Journal of Clinical Endocrinology and Metabolism 201095 4855-4863. (doi:10.1210/jc.2010-0982)

28 Bancos I, Hahner S, Tomlinson J \& Arlt W. Diagnosis and management of adrenal insufficiency. Lancet Diabetes Endocrinology 20153 216-226. (doi:10.1016/S2213-8587(14)70142-1)

29 Agha A, Tomlinson JW, Clark PM, Holder G \& Stewart PM. The long-term predictive accuracy of the short synacthen (corticotropin) stimulation test for assessment of the hypothalamic-pituitary-adrenal axis. Journal of Clinical Endocrinology and Metabolism 200691 43-47. (doi:10.1210/jc.2005-1131)

30 Clayton RN. Short Synacthen test versus insulin stress test for assessment of the hypothalamo (correction of hypothalmo)-pituitaryadrenal axis: controversy revisited. Clinical Endocrinology $1996 \mathbf{4 4}$ 147-149. (doi:10.1046/j.1365-2265.1996.666478.x) 
31 Kazlauskaite R, Evans AT, Villabona CV, Abdu TA, Ambrosi B, Atkinson $\mathrm{AB}$, Choi $\mathrm{CH}$, Clayton RN, Courtney $\mathrm{CH}$, Gonc EN et al. Corticotropin tests for hypothalamic-pituitary-adrenal insufficiency: a metaanalysis. Journal of Clinical Endocrinology and Metabolism 200893 4245-4253. (doi:10.1210/jc.2008-0710)

32 Dickstein G \& Saiegh L. Low-dose and high-dose adrenocorticotropin testing: indications and shortcomings. Current Opinion in Endocrinology, Diabetes, and Obesity 200815 244-249. (doi:10.1097/ MED.0b013e3282fdf16d)

33 Abdu TA, Elhadd TA, Neary R \& Clayton RN. Comparison of the low dose short synacthen test (1 microg), the conventional dose short synacthen test (250 microg), and the insulin tolerance test for assessment of the hypothalamo-pituitary-adrenal axis in patients with pituitary disease. Journal of Clinical Endocrinology and Metabolism 1999 84 838-843. (doi:10.1210/jc.84.3.838)

34 Wade M, Baid S, Calis K, Raff H, Sinaii N \& Nieman L. Technical details influence the diagnostic accuracy of the 1 microg ACTH stimulation test. European Journal of Endocrinology of the European Federation of Endocrine Societies 2010162 109-113. (doi:10.1530/EJE09-0746)

35 Murphy H, Livesey J, Espiner EA \& Donald RA. The low dose ACTH test - a further word of caution. Journal of Clinical Endocrinology and Metabolism 199883 712-713. (doi:10.1210/jcem.83.2.4587-1)

36 Krasowski MD, Drees D, Morris CS, Maakestad J, Blau JL \& Ekins S. Cross-reactivity of steroid hormone immunoassays: clinical significance and two-dimensional molecular similarity prediction. BMC Clinical Pathology 201414 33. (doi:10.1186/1472-6890-14-33)

37 Diagnostics R. Cortisol Kit Insert. Mannheim: Roche Diagnostics, 2010.

38 Czock D, Keller F, Rasche FM \& Haussler U. Pharmacokinetics and pharmacodynamics of systemically administered glucocorticoids. Clinical Pharmacokinetics 200544 61-98. (doi:10.2165/00003088200544010-00003)

39 Yates M, Watts RA, Swords F \& MacGregor AJ. Glucocorticoid withdrawal in polymyalgia rheumatica: the theory versus the practice. Clinical and Experimental Rheumatology 201735 1-2.

40 Van Ruiten HJ, Marini Bettolo C, Cheetham T, Eagle M, Lochmuller H, Straub V, Bushby K \& Guglieri M. Why are some patients with Duchenne muscular dystrophy dying young: an analysis of causes of death in North East England. European Journal of Paediatric Neurology 201620 904-909. (doi:10.1016/j.ejpn.2016.07.020)

41 Smans LC, Van der Valk ES, Hermus AR \& Zelissen PM. Incidence of adrenal crisis in patients with adrenal insufficiency. Clinical Endocrinology 201684 17-22. (doi:10.1111/cen.12865)

42 Hahner S, Spinnler C, Fassnacht M, Burger-Stritt S, Lang K, Milovanovic D, Beuschlein F, Willenberg HS, Quinkler M \& Allolio B. High incidence of adrenal crisis in educated patients with chronic adrenal insufficiency: a prospective study. Journal of Clinical Endocrinology and Metabolism 2015100 407-416. (doi:10.1210/ jc.2014-3191)

43 Quinkler M, Dahlqvist P, Husebye ES \& Kampe O. A European emergency card for adrenal insufficiency can save lives. European Journal of Internal Medicine 201526 75-76. (doi:10.1016/j.ejim.2014.11.006)

44 Quinkler M, Hahner S, Johannsson G \& Stewart PM. Saving lives of patients with adrenal insufficiency: a pan-European initiative? Clinical Endocrinology 201480 319-321. (doi:10.1111/cen.12378)

45 Sbardella E, Isidori AM, Woods CP, Argese N, Tomlinson JW, Shine B, Jafar-Mohammadi B \& Grossman AB. Baseline morning cortisol level as a predictor of pituitary-adrenal reserve: a comparison across three assays. Clinical Endocrinology 201786 177-184. (doi:10.1111/ cen.13232)

46 Woods CP, Argese N, Chapman M, Boot C, Webster R, Dabhi V, Grossman AB, Toogood AA, Arlt W, Stewart PM et al. Adrenal suppression in patients taking inhaled glucocorticoids is highly prevalent and management can be guided by morning cortisol. European Journal of Endocrinology of the European Federation of Endocrine Societies 2015173 633-642. (doi:10.1530/EJE-15-0608)

47 Grossman A, Johannsson G, Quinkler M \& Zelissen P. Therapy of endocrine disease: perspectives on the management of adrenal insufficiency: clinical insights from across Europe. European Journal of Endocrinology of the European Federation of Endocrine Societies 2013169 R165-R175. (doi:10.1530/EJE-13-0450)

48 Husebye ES, Allolio B, Arlt W, Badenhoop K, Bensing S, Betterle C, Falorni A, Gan EH, Hulting AL, Kasperlik-Zaluska A et al. Consensus statement on the diagnosis, treatment and follow-up of patients with primary adrenal insufficiency. Journal of Internal Medicine 2014275 104-115. (doi:10.1111/joim.12162)

Received 27 March 2017

Revised version received 16 June 2017

Accepted 4 July 2017 